Chirurg $2015 \cdot 86: 289$

DOI 10.1007/s00104-015-3000-6

Online publiziert: 6. März 2015

(c) Springer-Verlag Berlin Heidelberg 2015
P. Mroczkowski · C.J. Bruns

Klinik für Allgemein-, Viszeral- und Gefäßchirurgie, Universitätsklinik Magdeburg A.ö.R., Magdeburg

\section{Onkologisches Ergebnis beim pT1N0M0-Rektumkarzinom}

\section{Vergleich lokaler und radikaler Verfahren}

\section{Originalpublikation}

Kidane B, Chadi S, Kanters S et al (2015) Local resection compared with radical resection in the treatment of T1NOM0 rectal adenocarcinoma: a systematic review and meta-analysis. Dis Colon Rectum 58:122-140

\section{Hintergrund und Fragestellung}

Bei Frühkarzinomen des Rektums (T1N0M0) bilden transanale Resektionsoptionen eine attraktive Alternative $\mathrm{zu}$ Radikalverfahren. Das bessere funktionelle Ergebnis und die kleinere intraoperative Belastung sind jedoch kombiniert mit der Gefahr, durch belassene befallene Lymphknoten das onkologische Ergebnis zu verschlechtern. In dieser Metaanalyse wird die vorhandene Evidenz evaluiert.

\section{Methoden}

Als lokale Verfahren wurden TAE („transanal excision“), TEMS („transanal endoscopic microsurgery“) und TAMIS („transanal minimaly invasive surgery“) definiert, endoskopische Polypektomien wurden hier ausgeschlossen. $\mathrm{Zu}$ den radikalen Verfahren zählten transabdominale Prozeduren mit TME (,total mesorectal excision") wie tiefe anteriore Resektionen, Hartmann-Prozeduren sowie Rektumextirpationen. Als primäre Endpunkte wurden 5-Jahres-Gesamtüberleben, wichtige postoperative Komplikationen (definiert als „signifikanter langzeitiger Einfluss auf den Gesundheitszustand des Patienten") und die Notwendigkeit eines permanenten Stomas definiert. Als sekundäre Endpunkte wurden das 5 Jahre tumorfreie und krankheitsspezifische Überleben, die 5-Jahres-Lokalrezi- divrate und die perioperative Mortalität ausgewertet. Durchsucht wurden verfügbare Internetpublikationsdatenbanken sowie Kongressberichte.

\section{Ergebnisse}

Die Auswahlkriterien erfüllten 12 Observationsstudien und eine kontrollierte randomisierte Studie, die insgesamt $2855 \mathrm{~Pa}$ tienten umfassten. Die durchgeführte Metaanalyse zeigte, dass lokale Verfahren ein signifikant schlechteres 5-Jahres-Gesamtüberleben erbrachten (72 mehr Todesfälle pro 1000 Patienten, 95\%-Konfidenzintervall[CI] 30-120), die TEMSSubgruppe zeigte jedoch kein schlechteres Überleben. Lokale Verfahren zeigten eine niedrigere perioperative Mortalität (relatives Risiko [RR] 0,31; 95\%-CI 0,14-0,71), weniger postoperativer Komplikationen (RR 0,20; 95\%CI 0,10-0,41) und weniger Stomata (RR 0,17; 95\%-CI 0,09-0,30).

\section{Diskussion und Fazit}

Die gut recherchierte Metaanalyse bestätigt das große Dilemma der adäquaten chirurgischen Therapie beim Frühkarzinom des Rektums. Lokale Verfahren liefern einen klaren funktionellen Vorteil, dieser relativiert sich jedoch durch das schlechtere onkologische Ergebnis. Die Autoren schlussfolgern, dass dieses Phänomen durch eine Selektion der tiefer gelegenen Karzinome für ein lokales Verfahren resultiert, da diese an sich eine schlechtere Prognose haben. Die häufig verwendete Argumentation, dass die in situ belassenen Lymphknoten die Ursache für das onkologische Ergebnis darstellen [1], bringt hier keine Klärung, da bei den mikrochirurgischen Verfahren, die kein schlechteres onkologisches Ergebnis aufweisen, die Lymphknoten ebenfalls in situ belassen werden. Tatsächlich werden die transanalen Prozeduren im Vergleich zu den mikrochirurgischen Verfahren eher für die tiefer gelegenen Tumoren verwendet.

Eine wichtige Einschränkung der Aussagekraft liegt in dem ausgewählten langen Zeitraum (ab 1979). In den 35 Jahren gab es deutliche Änderungen der diagnostischen Methoden, sodass die Vergleichbarkeit der erhobenen Befunde nicht gesichert ist. Trotzdem müssen die Ergebnisse der Arbeit in der Entscheidungsfindung und in der Patientenaufklärung berücksichtigt werden. Lokale Verfahren bleiben weiterhin eine attraktive Option für die Patienten [2], das mit der Methode verbundene Risiko muss mit dem Patienten besprochen und in der engmaschigen Nachsorge berücksichtigt werden.

\section{Korrespondenzadresse}

\section{Prof. Dr. C.J. Bruns}

Klinik für Allgemein-, Viszeral- und Gefäßchirurgie, Universitätsklinik Magdeburg A.ö.R., Leipziger Str. 44, 39120 Magdeburg christiane.bruns@med.ovgu.de

Interessenkonflikt. P. Mroczkowski und C.J. Bruns geben an, dass kein Interessenkonflikt besteht.

\section{Literatur}

1. Kulu Y, Muller-Stich BP, Bruckner T et al (2014) Radical surgery with total mesorectal excision in patients with T1 rectal cancer. Ann Surg Oncol [Epub ahead of print]

2. Valsdottir EB, Yarandi SS, Marks JH et al (2014) Quality of life and fecal incontinence after transanal endoscopic microsurgery for benign and malignant rectal lesions. Surg Endosc 28:193-202 